\title{
SMARTPHONE AND COVID-19 CRISIS: HOW THE SMARTPHONE TECHNOLOGY IMPACTED LIFE UNDER COVID-19 PANDEMIC
}

\author{
Sarah Alowais. Robert Morrison University, sxast228@mail.rmu.edu
}

\begin{abstract}
Covid-19 affected life in many ways, and the challenges that humanity faced required different approaches to address them. Among the many technology-based solutions used to address these challenges, the Smartphone and its embedded technology played a significant role during this pandemic. With the fact that today many people use Smartphones, it proved to be a handy device for creating convenience and versatility in communication and information dissemination. Several issues of concern that relate to Information Systems were noted as discussed under the sections of Smartphones and Communication, Smartphone and Contact tracing, Smartphones and diagnostics, Smartphones and online businesses, and Smartphones as a risk of contamination. Data was collected from news websites and past research studies. Based on findings, the study's implications are outlined in later sections indicating areas that need further research and presents ideas on how the Smartphone could have been used in ways that are aligned with the ethical use of technology.
\end{abstract}

Keywords: Covid-19, Smartphone, e-commerce, Information Technology

\section{INTRODUCTION}

The year 2020 saw the Covid-19 pandemic devastate many sectors. The impact of this pandemic exposed critical areas of disaster management and preparedness in ways that IT was needed to meet some specific needs that resulted from the pandemic. This paper specifically delves into how the Smartphone contributed to the face of the problems that resulted from the Covid-19 crisis. The aim is to highlight issues related to the use of the Smartphone as an IT device and issues related to its use, productivity and ethical issues that are of interest to the field of Information Systems.

\section{BACKGROUND}

The Covid-19 pandemic became a new challenge facing humanity and has had a significant impact on society in different ways. The challenges introduced by the Covid19 pandemic were extensive, including diagnostics challenges, patient management challenges, information dissemination, and supporting business in the wake of extensive lockdowns and disrupted business processes due to the stay-at-home orders that were passed in many regions to curb the spread of this virus. The Smartphone is one of the devices that made a significant impact in addressing some of the pressing challenges introduced by the Covid-19 crisis. Concerning Information Systems, the Smartphone

\section{LITERATURE REVIEW}

The Smartphone has one of the devices that have become a handy IT device. Today, the Smartphone has extensive use in communication and access to information through the use of internet (Batista \& Gaglani, 2013). Smartphones have also found other uses across many industries in ways that are unique to the needs within these particular industries. Research by Engelbrecht, Booysen, Bruwer and Rooyen (2015) demonstrated that Smartphones could be used in vehicle-based monitoring systems, such as driving behavior and style recognition, accident detection, and road condition monitoring systems. In health care, there is a wide variety of Smartphone-compatible medical devices such as blood pressure cuffs, weight scales, and pulse oximeters that are being used to support home-based patient self-care (Batista \& Gaglani, 2013). Within the clinical setting, Smartphone-based apps and connections to other Smartphoneenabled devices have made it easier to collect clinically relevant data.

Concerning the use of Smartphones during the Covid-19 pandemic, not many studies had been done about COVID 19 pandemic as of Mid May 2020. However, several areas had come to attention in the few studies that had been done. One particular issue that came up and was reported across different news networks was the impact of fake news and 
misinformation on Covid-19. According to Washingtonpost, some ideas shared included drinking sanitizers, gargling bleach, injection with disinfectants as ways to treat Covid-19, which were all dismissed as fake news and misinformation (Rosenbloom, 2020). The Hydrolochloroquine drug used to treat Malaria also received a global hype as a medication that was having positive impacts on Covid-19 patient's but was later dismissed after trials and monitoring of patients (Boulware, Pullen \& Nascene, 2020). This information was readily available and mainly shared through websites and social media sites.

Duraisamy, Rathinaswamy, \& Sengottaiyan (2020) conducted a qualitative study to assess whether social media was informing or misinforming the public concerning the Covid-19 pandemic a survey of 30 social media users. Their findings indicated that while most information acquired by social media users was accurate, there were some elements of misinformation in more than half of the surveyed persons. They also showed that most of the users tried to query the reliability of the information, but it was challenging in some aspects of assessment.

Concerning challenges that came about with the Covid-19 pandemic, non-online businesses were affected, with the transport industry being the worst hit (Dhar, 2020). Interestingly, some business boomed at the same time, particularly the e-commerce sites that handle basic needs commodities and other essentials. Saunders (2020) noted that services that help in virtual conferences also received wider usage, noting that Zoom emerged as one of the biggest winners in this pandemic. Socially, livelihoods were largely affected, which necessitated many people to look for entertainment and socialize during COVID 19 pandemic during the widespread lockdowns and stay-at-home orders. Many of these relied on technology. To learners, online learning was necessary, and online classroom technology also gained higher usage (Saunders, 2020).

Based on these areas that have already been studied, this research study focuses on the position and role of Smartphones in life under the Covid-19 crisis. This study highlights elements related to Smartphone technology and the use of Smartphones and how it bridged different gaps that resulted from the Covid-19 pandemic. Through ideas presented in this paper, areas that need further research will be highlighted based on the extent of the impact of smartphones in life under the Covid-19 crisis from a technological point of view and the impact of technology on social and economic perspectives. The issues raised are relevant to Information Systems by highlighting how Smartphones, as one of the IoTs has significant value in pandemic response and management strategies, both as a communication device and in utilizing its embedded technologies.

\section{RESEARCH METHODOLOGY}

This study takes a qualitative approach to secondary sources. The secondary sources used were credible news websites covering the events related to the Covid-19 crisis and the life under the crisis, as well as peer-reviewed journal articles. The keyword search method was used to identify sources that related to Covid-19 and Smartphones. A total of 12 sources were identified as marching the relevance criteria for this study. These sources were a combination of peerreviewed journal articles and news articles from reputable sources.

The research questions for this study were:

i) What specific ways did Smartphones affect life during the Covid-19 pandemic?

ii) What were specific issues of concern relating to Information Systems notable in the way the Smartphone was during the Covid-19 crisis?

\section{FINDINGS}

The main areas that are identified as critical areas of analysis of the role of Smartphones during the Covid-19 crisis are Smartphones and Communication, smartphones and Contact tracing, Smartphones and diagnostics, Smartphones and online businesses, and Smartphones as a risk of contamination as a handheld device.

\section{Smartphones and Communication During Covid-19 Crisis}

As of late 2019, the number of Smartphone users in the world was 3.5 billion, which means that about $45.04 \%$ of the world's population owns a Smartphone (Statista, 2020). The Smartphone has become a handy gadget because it provides a means of wireless communication either through voice or via text. It also provides a platform to access the 


\section{Issues in Information Systems}

Volume 21, Issue 4, pp. 193-201, 2020

internet on the go. In many other ways, it has become an entertainment gadget and a tool to capture moments that are worth keeping memories or sharing media files.

In the wake of the Covid-19 crisis, the Smartphone as a communication device played very significant roles. Since the novel Coronavirus was reported, information was widely shared about the new disease in China through social media (Wajahat, 2020). The information was very important because of the rest of the world. They did not have much information about the signs and symptoms of this novel Coronavirus, and how it affected persons infected. According to search statistics provided by Google Inc, the Coronavirus was the most searched topic globally on its search engines, with its peak being in March 2020 through April 2020. Of this search history, about 70\% came from mobile devices, which was a clear indication that the Smartphone was a critical tool in getting information about Covid-19 (Wajahat, 2020). The most common search phrases were: What is Coronavirus, Signs and symptoms of Coronavirus, is coronavirus airborne, how did Coronavirus start, coronavirus vaccine and when Coronavirus will end. Based on these statistics, they show that people needed to access information about the novel Coronavirus. Comparing this situation with past pandemics before the Smartphones came, the situation must have been very different. For instance, during the 1918 influenza pandemic that was the most severe in recent history, information was mainly disseminated through mainstream media. With the coming of the internet and internet-enabled mobile phones, accessing information access became much easier and convenient, as evidenced by the way people searched for information related to Covid- 19 .

Social media has been critical in addressing some of the challenges introduced by the Covid-19 crisis. In addition to Smartphones being the main avenues through which people accessed and shared information about positive behavior change on matters such as staying safe and protecting others, they were also used to address some of the negative issues related to Covid-19. For instance, there were reports of some discrimination of persons who were thought to be from hotspot areas of the Covid-19 or brutality towards civilians when enforcing civil lockdowns. Such incidents were often captured and widely shared through social networking apps on Smartphones and similar devices. Smartphones provide a camera on the go has made virtually anyone with a Smartphone, a journalist on the go. This idea is not a farfetched since the basic definition of a journalist is a person who collects, writes, or distributes news or other current information to the public. During the crisis, much of this was done using Smartphones by ordinary persons and shared through the captured information through social media.

There were many of these instances where Smartphones were used for journalistic purposes and bringing to attention some of the issues that needed to be addressed when dealing with the Covid-19 crisis. For example, many incidents were captured in videos and photos by civilians indicating the law enforcement agencies using excessive force to enforce the guidelines that authorities had outlined to curb the spread of Covid-19. The countries where this abuse was evident based on videos and photos that were shared included India, Philippines, Kenya, Rwanda, South Africa, Uganda and Brazil (Dahir \& Cave, 2020). In some cases, the spreading and reaction towards these incidents led to revisions on how the law enforcement agencies were supposed to enforce this curfew, lockdown and social distancing measures that had been stipulated.

The Smartphone was also used to disseminate information about the challenges that people were facing during the pandemic. For example, videos and photos were shared about charity works to the poor in helping them get food. In others, they highlighted cases of discrimination, particularly against the Asians in the US and other parts of the world, because of the claim that Covid-19 originated in China. One of the most disturbing profiling scenes that were captured and shared by onlookers was an Asian woman in New York who was physically abused and bullied (Human Rights Watch, 2020). This incident, along with others, brought to light some of the new developments that the Covid-19 pandemic was creating among the people that needed immediate action to stop discrimination and profiling of some innocent persons.

Further, Smartphones became very handy devices to facilitate communication during the stay at home times. This kind of communication was enforced by the technologies that are emended into Smartphones. The most notable one was that $\mathrm{t}$ many people started using the teleconferencing App called Zoom for their teleconferencing needs, particularly teachers and students in online class setups (Saunders, 2020). The Zoom App uses the camera of a device to facilitate video conferencing. Although not exclusively used on Smartphones, this application was one of the technologies that helped overcome some of the challenges that had been introduced to the Covid-19 crisis. Other Smartphone-based applications that were used to aid in video conferencing in making virtual face to face communication during times that people were required to stay at home included Whatsapp and Facebook App, which 
recorded much higher usage on their video calling features (Saunders, 2020). Other Apps that gained increased usage are as summarized in Fig 1 below. These technologies embedded in Smartphones helped address communication challenges that came with the Covid-19 crisis and the guidelines that had been enforced to help curb the spread of the disease.

\section{Daily app sessions for popular remote work apps}

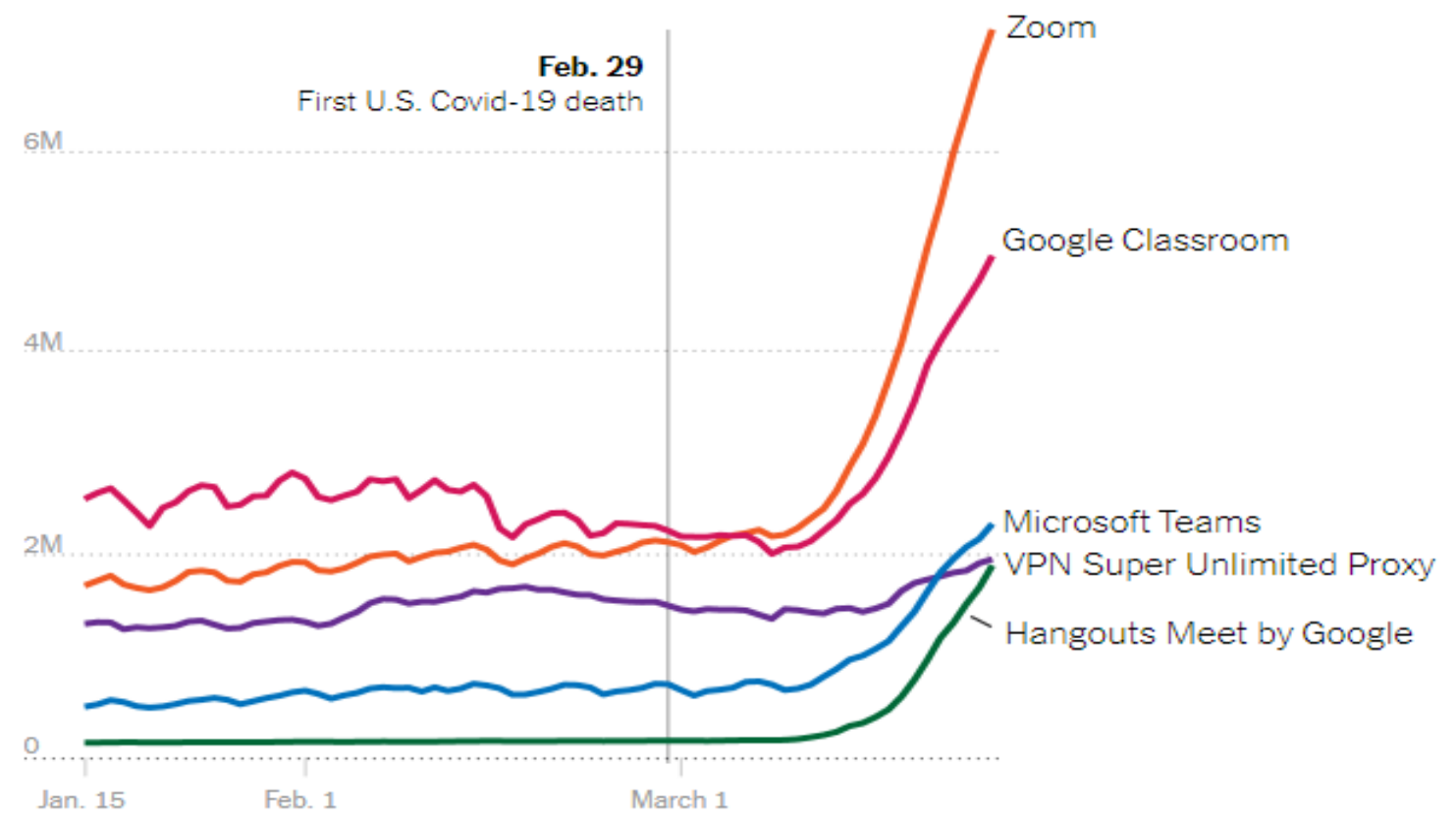

App popularity according to iOS App Store rankings on March 16-18. - Source: Apptopia

Figure 1. Smartphone Apps that gained higher usage during Covid-19 Pandemic Source: Apptopia.com

One major challenge that is of interest to how the Smartphones were being used to facilitate virtual face to face communication was the hacking of conference sessions, with a particular note on the Zoom application, which was the most widely used teleconferencing app (Saunders, 2020). Zoom, being cloud-based video conferencing service used to virtually meet with others either by video or audio-only or both conducting live chats and lets users record these sessions to view later, was a particular target (Saunders, 2020).

The security issue that arose may partly be linked to how mobile devices access the internet. One major concern was on security and privacy, after several instances of having intruders doing Zoom chats and conferences. According to the developers of this App, this new attack on Zoom, which became known as Zoombombing, was because PMIs are always accessible using the same ID or meeting link. Anyone can join unless they're properly secured (Saunders, 2020). This design was the main reason why intruders were able to join sessions. With Zoom and Smartphones, there were some suggestions that the Apps were less secure than the Web interfaces of Zoom. As such, it was clear that the Zoom App had introduced a vulnerability to the end-users.

Many other Apps were developed during the COVID 19 crisis to facilitate communication and dispatching or collecting data related to COVID 19 tracing. Just like Zoom, there is a significant need to research any ways in which these Apps may have led to some security issues that may already have been noted or not yet noted. Thus, security issues of Apps that gained high usage during the Covid19 pandemic need to be studied to determine any areas of security that maybe need to be improved or addressed concerning data security and privacy. 


\section{Issues in Information Systems}

Volume 21, Issue 4, pp. 193-201, 2020

\section{Smartphones and Contact Tracing}

In consideration of how contact tracing has been carried out during the Covid-19 pandemic, several steps were involved in making contact tracing an effective measure of controlling the spread and containing the virus from spreading further. In the absence of comprehensive testing capabilities, contact tracing plays a vital role in identifying possible and probable cases from the contacts with the confirmed cases of Coronavirus (Abeler, Backer, Buermeyer, \& Zillessen, 2020). Smartphones were very helpful in this management exercise. One step that was taken by the use of Smartphones was identifying contacts, and this is done by outlining the people that one has been in contact with before two days or within the 14 days of the onset of the illness (Abeler, Bäcker, Buermeyer, \& Zillessen, 2020). In defining the contacts, one is suspected, and the tracing starts when the suspect has been within one meter with the COVID-19 case or in case of direct contact with the infected person. Defining contacts can also be outlined if a person takes care of Covid-19 diseased person without the proper attires for protective and preventive measures. And if the confirmed cases are asymptomatic, then contacts should be treated as the same as the symptomatic cases and be quarantined for 14 days or be kept in self-isolation for the same 14 days. The possible contacts are defined through the use of smartphones, and now the second step comes in where the contacts are identified. Smartphones have GPS trackers that can show a suspect location, and the surveillance teams go to the place and get the person and do the necessary testing. If need be, then applying the necessary precaution measures such as initiating a lockdown is upheld. Detailed case investigation and the interview with the Covid-19 patient is needed. Identification of contacts based on the settings such as the households or the community closed settings such as prisons, healthcare settings, public or shared transport and gatherings. After that, a direct interview can be done with the patient through the use of smartphones to provide for the contact details of the person who has been in contact within recent days (Abeler, Bäcker, Buermeyer, \& Zillessen, 2020). Informing contacts is the third step, and the tracing team prepares a list of suspected contacts, and they get contacted via phones. If they meet the provided details, they are addressed on the quarantine measures and how to look out for the known symptoms such as fever. Managing and monitoring contacts are the final step. It is effective through the provision of quarantines, filling of contact tracing forms, regular communications, physical distancing, self-reporting and the capturing of the data using the capturing tools such as demography information, contact information and daily follow-ups of symptoms.

\section{Smartphones and Clinical Diagnostics}

According to Biospace, "NSF-funded Smartphone COVID-19 diagnostic test could put testing in the hands of the public" (Dutton, 2020). This approach is just one of the kinds of approaches that many medical research labs have taken to make diagnosis more cost-effective and convenient for the target populations. One of the major issues that were noted during the crisis was the capacity to test COVID 19 was a critical factor in the management of the pandemic because positive cases could be identified early, and necessary steps are undertaken such as quarantining the identified positive subjects and performing contact tracing. In many cases, testing resources were heavily constrained mainly because the tests had to be performed by a medical professional conversant with sample collection procedures and testing the collected sample. The process also required a high volume of disposable consumables that would mean that a lot of resources were needed. Some facilities, such in less developed countries, experienced significant constrain in testing resources. In other cases, logistical challenges involving the transport of the testing kits and the manufacturer were also common challenges limiting the efficiency in testing the COVID 19. This aspect was a significant challenge because, in the face of a pandemic, testing kits for the disease of concern are needed in many parts of the globe.

The potential indicated in the research that was geared towards making the Smartphones become a revolutionary, the new diagnostic device for COVID 19 was a very good idea. This approach's goal was to provide an easy and low-cost way to monitor and diagnose a large population without the need for special equipment or clinicians' involvement. With the achievements made as of early June in development of this kind of Smartphone-based Covid-19 testing kits, several Smartphone-based COVID 19 testing prototypes were in advanced stages of development with very positive prospects.

The interesting part of the Smartphone Apps under development and testing is that they had a different approach to running their diagnostic. One App being developed by Sanofi and Luminostics was designed to use a glow-in-thedark nanoparticle that could be picked up by a Smartphone's camera and deliver results in 30 minutes without needing a medical professional (Cogley, Boland \& Hoggins, 2020). For this idea to work, there would be nanoparticles packaged as self-testing kits for sale in local outlets where interested candidates would buy the kit, collect the sample 
themselves using a swab and analyze it using a Smartphone App that uses a combination of both the camera of the Smartphone and Artificial Intelligence (AI). This approach would make testing easier and cheaper to administer, especially at times of a pandemic.

Another Smartphone App that was in advanced stages of testing its prototypes was an App called Coughid App developed by scientists at the Swiss university (Cogley, Boland \& Hoggins, 2020). The approach of this App is to use artificial intelligence to listen to people's cough and determine whether they sound like a Coronavirus patient. This idea was developed on information from reports from doctors that Covid-19 patients had a cough with a distinctive sound of a chirping intake of breath at the end differing from other illnesses (Cogley, Boland \& Hoggins, 2020). As such, they were capturing data related to the coughs was one of the prospects that could be used to identify if a person may have contracted Covid19.

Even though by early June 2020 no Smartphone-based Covid-19 testing had been launched yet, findings from other ways in which Smartphones have been used to diagnosis some diseases offer prospects that a Smartphone-based app for Covid-19 was not a farfetched idea, and explains the reason such projects have been pursued or received significant funding. As such, the Covid-19 pandemic highlighted the value that Smartphones might have concerning facilitating easier, cheaper and more convenient disease diagnostics, especially at times of pandemics when critical resources needed for testing may be limited.

\section{Smartphones and Online Businesses}

The COVID 19 pandemic changed the business environment significantly. One major impact was that extensive lockdowns and self-isolation as measures enforced to curb the spread of the infection made many people stay at home. This lifestyle meant that many of them never went to shops as often as before the pandemic. There was much close up of small businesses because of the inadequate flow of customers as they largely stayed at home. The only promising avenue that remained was the online selling of products and services.

The Smartphone and its embedded technology came in handy for this need. According to statistics on new apps being used for commerce during the Covid-19 crisis, there was a surge in enrollment of these apps in App stores starting in March 2020 when extensive lockdowns started (Marketplace.org, 2020). Another dimension showed a marked increase in the use of some e-commerce apps that sell some basic commodities. For instance, the Apps of meal delivery business such as Grubhub, Doordash, Uber Eats and Postmates recorded a new high in usage in the US during the COVID crisis (Marketplace.org, 2020). This App usage is an indication that these apps that are mainly used on Smartphones bridged a significant online business gap by providing a platform to connect the seller and the consumer easily, conveniently and in a very cost-effective way.

\section{Smartphone and Risk of Contamination}

During the Covid-19 pandemic, handwashing and sanitizing hands and surfaces became a new norm to combat the spread of the virus. The Smartphone, however, presented a major challenge in avoiding contamination because of being a handheld gadget that may not always be resistant to liquids (Panigrahi, Pathak \& Kumar, 2020). Although many Smartphones are water-resistant, there is a significant number that is not water-resistant. The design of Smartphones traditionally has not been designed to be sanitized. This aspect was a significant risk factor to many individuals because of the dilemma on how to handle Smartphones when they were likely to be contaminated. Yet, there was no standard safe way of sanitizing some of these phones endorsed by manufacturers or other groups.

Mobile phones and hygiene were noted to be part of the neglected areas. In parts studies on the prevalence of contaminants on mobile phones, about $68 \%$ of mobile phones have been found to contain microbes (Panigrahi, Pathak \& Kumar, 2020). In times of pandemics of highly contagious microbes such as the Covid-19 virus, such contamination is critical because people have been found to touch their phones an average for three hours every day, with super-users touching phones more than 5,000 times a day. Unlike hands, mobile devices are not regularly washed. To many users, there was a dilemma of how to handle mobile phones after visiting grocery stores. They were not sure how to sanitize their phones, and subsequently, handling an un-sanitized phone after sanitizing hands was beating the logic of sanitizing the hands. As part of recommendations, experts indicated that surface decontamination with $70 \%$ alcohol would sanitize mobile phones (Panigrahi, Pathak \& Kumar, 2020). However, this message was not widely passed, as 
did the practice of handwashing during the Covid-19 crisis. It is most likely that there were many cases where the source of contamination that eventually led to infection was from the use of mobile phones.

\section{DISCUSSION OF FINDINGS}

As noted from this analysis of the position of Smartphones during the 2020 Covid-19 pandemic, several issues are worth noting. First, despite the Smartphones being effective communication devices, pandemic situations may introduce some vulnerabilities to users such as misinformation and fake news. Being a major avenue that many people currently access information, there is needed to separate facts from fads and fake news to safeguard communities. For example, there were many fatalities related to people drinking sanitizers simply from information being shared, and Smartphones bears the significant load of accessing information during pandemics. Campaigns to address these vulnerabilities related to access to misleading information was needed.

Secondly, as much as Smartphones and their embedded technology may serve as good sources of gathering personal data, as done during contact tracing, data privacy, and ethical use of acquired data need to be considered. This issue was also noted to be a serious issue of concern on how the Smartphones were as journalistic tools on the go. Many people captured events related to COVID 19 crisis on their Smartphones and shared them through the same devices using social media sites. This usage had positive impacts in some ways, such as bringing to light some bad occurrences such as police brutality during lockdown and curfew enforcement. Still, at other times, it may have been a breach of privacy of some individuals. A balance is therefore needed, which can be enforced through data security and privacy regulations.

Thirdly, the Smartphone is a critical part of some e-commerce businesses. From statistics, most of the meals were ordered through Smartphone Apps. Many sites also came up to supplement the brick and mortar stores. This trend highlights the value that e-commerce has in today's dynamic business environment, and how ordinary technologies like Smartphone and its embedded technologies facilitates the goals of e-commerce.

Fourthly, the design of Smartphones needs to take into account how it can be effectively disinfected. Even in the absence of pandemics, the mobile phone may be a reservoir of disease-causing microbes. Manufacturers, therefore, need to start offering guidelines on how users can disinfect their phones when needed. In the times of pandemics associated with highly contagious microbes, awareness campaigns will also be needed in the future because not much was geared towards letting the people know how to disinfect their mobile phones. Yet, there was an extensive campaign to promote frequent handwashing practices.

\section{IMPLICATIONS FOR RESEARCH}

From the areas of interest highlighted in this research paper, it is recommended that areas that related to data security and privacy need further study. This is because at the time of composing this paper, little had been studied, and a key source of information acquired was from news. Thus, primary sources through surveys and questions of persons affected by some of the data security and privacy issues covered would highlight other valuable information related to this subject. This method was not used because it was beyond the scope of this research paper. Based on the findings and recommendations, this research will help policy development, particularly in areas that need end-user education and the enlighten of Smartphone users during pandemics. This is because pandemics are associated with the hype of searching for information about the health condition of concern. The internet, which is largely accessed by a vast majority through smartphones, is often a source of misinformation and unverified information. For agencies that would need to use Smartphones to collect data about its holders, new policies need to be developed on guidelines of usage of the collected data to prevent data and privacy breaches.

\section{CONCLUSION}

From this analysis, it highlights the position that Smartphones had in Covid-19 crisis. This was considered because of the extensive ways in which Smartphones transformed life under COVID 19 crisis. Important areas of consideration were Smartphones and Communication in COVID Crisis, Smartphone and Contact tracing, Smartphones and diagnostics, Smartphones and online businesses, and Smartphones as a risk of contamination. From the information gathered, it was clear that while the Smartphone bridged significant gasp during the crisis, there are areas that need to 
be addressed, as highlighted in the recommendations section. This information will be important in case of another pandemic based on the lessons learned from the role of the Smartphone during the COVID 19 crisis.

\section{REFERENCES}

Abeler J, Bäcker M, Buermeyer U, Zillessen H. (2020). Contact Tracing and Data Protection Can Go Together. JMIR Mhealth Uhealth. 2020;8(4):e19359. Published 2020 Apr 20. doi:10.2196/19359

Batista, M., \& Gaglani, S. (2013). The Future of Smartphones in Health Care. AMA Journal Of Ethics, 15(11). doi: 10.1001/virtualmentor.2013.15.11.stas1-1311

Boulware, D., Pullen, M., \& Nascene, A. (2020). A Randomized Trial of Hydroxychloroquine as Post-exposure Prophylaxis for Covid-19. New England Journal of Medicine, 1(4). doi: 10.1056/nejmoa2016638

Cogley, M., Boland, H., \& Hoggins, T. (2020). Sanofi working on Corona virus test that can be done on a Smartphone. Retrieved Jun 9 2020, from https://www.telegraph.co.uk/technology/2020/04/17/sanofi-workingcoronavirus-test-can-done-smartphone/

Dahir, A., \& Cave, D. (2020). How Far Should Police Go in Enforcing Coronavirus Lockdowns? Retrieved Jun 3 2020, from https://www.nytimes.com/2020/04/02/world/australia/coronavirus-police-lockdowns.html

Dhar, Ravi. (2020). COVID-19 Challenges facing the Media. 10.13140/RG.2.2.15636.04489.

Duraisamy, Brindha, Rathinaswamy, Jayaseelan \& Sengottaiyan, Kadeswaran. (2020). Social Media Reigned by Information or Misinformation about COVID-19: A Phenomenological Study. SSRN Electronic Journal. 09. 585-602. 10.2139/ssrn.3596058.

Dutton, G. (2020). NSF-funded Smartphone COVID-19 Diagnostic Test Could Put Testing in the Hands of the Public. Retrieved Jun 6 2020, from https://www.biospace.com/article/nsf-funded-smartphone-covid-19-dx-putstesting-in-the-hands-of-the-public/

Engelbrecht, J., Booysen, M., Bruwer, F., \& van Rooyen, G. (2015). Survey of smartphone-based sensing in vehicles for intelligent transportation system applications. IET Intelligent Transport Systems, 9(10). doi: 10.1049/ietits.2014.0248

Human Rights Watch. (2020). Covid-19 Fueling Anti-Asian Racism and Xenophobia Worldwide. Retrieved Jun 6 2020, from https://www.hrw.org/news/2020/05/12/covid-19-fueling-anti-asian-racism-and-xenophobiaworldwide

Marketplace.org. (2020). Restaurants hit by COVID-19 say delete delivery apps - Marketplace. Retrieved Jun 5 2020, from https://www.marketplace.org/2020/04/16/restaurants-hit-covid19-say-delete-delivery-apps/

Panigrahi, S., Pathak, V., \& Kumar, M. (2020). Covid-19 and mobile phone hygiene in healthcare settings. BMJ Global Health, 5(4), e002505. doi: 10.1136/bmjgh-2020-002505

Rosenbloom, C. (2020). Garlic and bleach won't cure Coronavirus. How such myths originated - and why they're wrong.. Washington Post. Retrieved Jun 2 2020, from https://www.washingtonpost.com/lifestyle/wellness/cures-coronavirus-myths-garlic-silverbleach/2020/03/09/04ec9fa6-6234-11ea-845d-e35b0234b136_story.html.

Saunders, J. (2020). A Meteoric Rise \& Fall: The Impact of COVID-19 on Zoom - DevPro Journal. DevPro Journal. Retrieved Jun 8 2020, from https://www.devprojournal.com/technology-trends/a-meteoric-rise-fall-theimpact-of-covid-19-on-zoom/. 


\section{Issues in Information Systems}

Volume 21, Issue 4, pp. 193-201, 2020

Statista. (2020). Smartphone users worldwide 2020. Retrieved Jun 10 2020, from https://www.statista.com/statistics/330695/number-of-smartphone-users-worldwide/

Wajahat, H. (2020). Role of Social Media in COVID-19 Pandemic. The International Journal of Frontier Sciences. 4. 10.37978/tijfs.v4i2.144. 